\title{
Tissue Optical Clearing in the Ultraviolet for Clinical Use in Dentistry to Optimize the Treatment of Chronic Recurrent Aphthous Stomatitis
}

\author{
Alexey A. Selifonov ${ }^{1,2 *}$ and Valery V. Tuchin ${ }^{1,3,4}$ \\ ${ }^{1}$ Saratov State University, 83 Astrakhanskaya str., Saratov 410012, Russia \\ ${ }^{2}$ Children's Polyclinic No.3 of the Children's Infectious Diseases Clinical Hospital No.5, 40 Moscow str., Saratov 410031, \\ Russia \\ ${ }^{3}$ National Research Tomsk State University, 36 Lenin Avenue, Tomsk 634050, Russia \\ ${ }^{4}$ Institute of Precision Mechanics and Control of the Russian Academy of Sciences, 24 Rabochaya str., Saratov 410028, \\ Russia \\ *e-mail: peshka029@gmail.com
}

\begin{abstract}
The immersion optical clearing (OC) treatment with a highly concentrated glycerol solution has induced three new tissue windows in the UV spectral range of gingival tissues - from 200 to $250 \mathrm{~nm}$, from 250 to $300 \mathrm{~nm}$ and from 300 to $400 \mathrm{~nm}$. By combining the immersion OC technique in human tissues with UV-spectroscopy, it was possible to verify and study the major OC mechanisms - tissue dehydration and refractive index matching, and that the OC efficiency is higher in the deep-UV than in the visible-NIR range. Since all biological tissues present high scattering in the UV range, the presented technology, which basically reduces the strong light scattering in the UV range, has a broad application area in medicine. The effectiveness of the developed technology combining UV phototherapy and OC in application to treatment of aphthous recurrent stomatitis in children was demonstrated. (C) 2020 Journal of Biomedical Photonics \& Engineering.
\end{abstract}

Keywords: immersion optical clearing; UV spectrum; human gingiva; aphthous recurrent stomatitis.

Paper \#3394 received 15 Nov 2020; revised manuscript received 20 Dec 2020; accepted for publication 22 Dec 2020; published online 31 Dec 2020. doi: 10.18287/JBPE20.06.040301.

\section{Introduction}

When radiation interacts with biological tissues, such effects as absorption, reflection and scattering are observed that, to varying degrees, make a significant contribution to the diagnosis of diseases, the study of healthy and pathological biological tissues, the development of photothermal and light therapy procedures, etc [1]. Biophysical and photochemical studies have shown that UV radiation causes ionization of molecules and the destruction of covalent bonds in biological tissues; visible radiation - causes their electronic excitation; and infrared radiation is capable of changing oscillatory processes in biological molecules. It was found that short-wave UV radiation causes lethal mutations in microorganisms and has a bactericidal effect; medium-wave UV radiation induces the synthesis of provitamin D and activates biogenic amines in the irradiated tissues; long-wave UV radiation induces the transport of melanin granules and inhibits the pathological proliferation of cells of pre-sensitized biological tissue [2].

Optical imaging of deep tissue layers is used to identify malignant neoplasms in the early stages by applying visible and NIR imaging systems, including optical coherence tomography (OCT), fluorescence, Raman, and multiphoton microscopies, fiber optic technologies for delivering radiation to pathological areas and back [3].

The main source of light scattering in biological tissues is the inhomogeneity of the refractive index due to the difference in its values for the components of biological tissues, i.e. mitochondria, nuclei, other organelles and cell cytoplasm; or for interstitial fluid and 
structural elements of connective (fibrous) tissue (collagen and elastin fibers) [4].

Intravital noninvasive high-resolution imaging of the structure of the tissues of the oral cavity using OCT in its various modes demonstrates excellent ability to detect and diagnose precancer, early cancer, dysplasia and malignancy of the mucous membrane epithelium [5-8]. The use of color spaces, known as CIELAB, allowed one to evaluate the color parameters of oral tissues in normal and pathological conditions [9-12].

To improve the penetration of radiation deep into biological tissues, a decrease in light scattering is required, which can be achieved by the optical clearing (OC) method [4, 13]. For immersion OC of biological tissues, both hyperosmotic solutions, such as glucose, sorbitol, glycerol, polyethylene glycol, propylene glycol, dimethyl sulfoxide, and solutions with normal osmolarity, such as X-ray contrast agent iohexol and iodixanol, are used. By using a hydrophilic optically transparent vinyl polysiloxane (VPS) impression material used in vivo for dental and gingiva impressions as an OC agent (OCA), the visibility of subsurface occlusal lesions and dentin-enamel compounds in the foci of demineralization was improved using reflectance spectroscopy [14]. The presence of pathological lesions was confirmed using polarized light microscopy and microradiography.

The strong scattering of biological tissues in the UV prevents the widespread use of UV technologies in medicine, in particular, in dentistry. Therefore, the aim of this study was to investigate the possibility of suppressing strong light scattering in the UV using suitable OCAs, transparent in the UV. Also, to prove the effectiveness of the developed OC technology in combination with UV phototherapy for treatment of aphthous recurrent stomatitis in children.

\section{Methodology}

\subsection{Ex vivo study}

For ex vivo study, pig alveolar gingival samples from the anterior part of the upper and lower jaws of freshly slaughtered farm pigs were excised just prior to measurements. The thickness of the samples was measured with a Micrometer MKTs-25-0.001 (China) placed between two glass slides; the measurement accuracy was $\pm 10 \mu \mathrm{m}$. The measurements were taken at five points, and then the values were averaged. The thickness of the cut of the gingival mucosa is on average $(0.59 \pm 0.08) \mathrm{mm}$. To measure the total transmittance and diffuse reflectance of tissue samples in the spectral range of 200-800 nm, a Shimadzu UV-2550 double-beam spectrophotometer (Japan) with an integrating sphere was used. The radiation source was a halogen lamp with radiation filtering in the investigated spectral range. The limiting resolution of the spectrometer was $0.1 \mathrm{~nm}$. The spectra were normalized before measurements using the $\mathrm{BaSO}_{4}$ reference reflector, which has suitable UV properties [15]. All measurements were carried out at room temperature $\left(\sim 25^{\circ} \mathrm{C}\right)$ and normal atmospheric pressure. Each sample of the tissue under study was fixed in a special frame with a $0.5 \times 0.5 \mathrm{~cm}^{2}$ window in a quartz cuvette so that the tissue sample was pressed against the wall of the cuvette and subjected to optical measurement. To measure the total transmission spectra (TTS), a quartz cuvette with a tissue sample was placed directly in front of the integrating sphere, collecting all radiation that passed through the tissue sample. The diameter of the light beam incident on the sample was $3 \mathrm{~mm}$.

Highly concentrated glycerol aqueous solution $87.5 \%$ (Russia) was used as an immersion agent. Glycerol $\left(\mathrm{C}_{3} \mathrm{H}_{5}(\mathrm{OH})_{3}\right)$ is a colorless liquid with extremely low absorption in the UV to visible and NIR (from $150 \mathrm{~nm}$ to $1400 \mathrm{~nm}$ [16]. It is a representative of trihydric alcohols with a molecular weight of $\mathrm{M}=92.1 \mathrm{~g} / \mathrm{mol}$, viscous, hygroscopic, non-toxic and odorless liquid.

An important parameter for quantifying the achieved tissue transparency is the OC efficiency, which is defined as [4]:

$$
Q(T)=\frac{T(t)-T(t=0)}{T(t=0)} \times 100 \%,
$$

where $T(t=0)$ is the tissue sample transmittance at the initial moment of time, $T(t)$ is the transmittance at the moment $t$.

\subsection{Clinical study in vivo}

The study analyzed the results of treatment of 120 patients with chronic recurrent aphthous stomatitis (CRAS). In each group, 40 volunteers with an established diagnosis of CRAS were selected, 20 boys and 20 girls in each group: group I (main); group II (comparison); group III (control). Standard treatment included detection and elimination of irritants during a dental examination: sharp edges of teeth, thorough debridement of the oral cavity if necessary; with a sharp soreness of aft application with anesthetic agents based on lidocaine $1 \%$ (novocaine $1 \%$ ) applied to a sterile bandage; removal of necrotic tissues, if any, using the proteolytic enzyme trypsin; antiseptic treatment with lugol on glycerol basis (aqueous solution of iodine $(0.01 \mathrm{w} / \mathrm{w})$, potassium iodide $(0.02 \mathrm{w} / \mathrm{w})$ glycerol $(0.94 \mathrm{w} / \mathrm{w})$ and water $(0.03 \mathrm{w} / \mathrm{w}))$; epithelial therapy in the form of rosehip oil applications to a sterile bandage and desensitizing therapy.

Group I (main) received, in addition to standard treatment, the proposed method of phototherapy with the use of OC with highly concentrated glycerol solution. Once per day for 6-12 days, a phototherapy procedure was performed: an application of glycerol solution (a pharmaceutical preparation without dilution) was preliminarily done for $10 \mathrm{~min}$ using a sterile bandage on the oral cavity, covering the erosive and ulcerative lesions; then the mouth was rinsed with warm boiled water and the entire oral cavity was irradiated during $12 \mathrm{~min}$ through a special tube with diameter $15 \mathrm{~mm}$ with a power density on the tube output of 
$2.0 \mathrm{~W} / \mathrm{m}^{2}$ using a commercially available device OUFv-02 (Russia) (230-400 nm).

Group II (comparison) received, in addition to standard treatment, a similar phototherapy as for Group I, but without preliminary OC with glycerol solution.

Group III (control) received standard treatment with lugol, for this: 4-6 times a day, lugol was applied to the affected areas oral cavity (with preliminary rinsing) until the aphthae disappeared.

The recommendations of the Ethical Committee were received from the Saratov State Medical University named after V.I. Razumovsky, protocol No. 8 dated 07.04.2020. All volunteers included in the study signed a voluntary consent to participate in the study.

\section{Results and Discussion}

\subsection{Ex vivo study}

From an optical point of view, the tissue of the gingival mucosa may be classified as irregular fibrous tissue, which is optically turbid. After immersion of gingival tissue samples in $87.5 \%$ glycerol solution, the total transmittance (2) increase (Fig. 1a, 1b) in relation to the original samples (1), which indicates a decrease of light scattering by gingival samples as a result of OC.

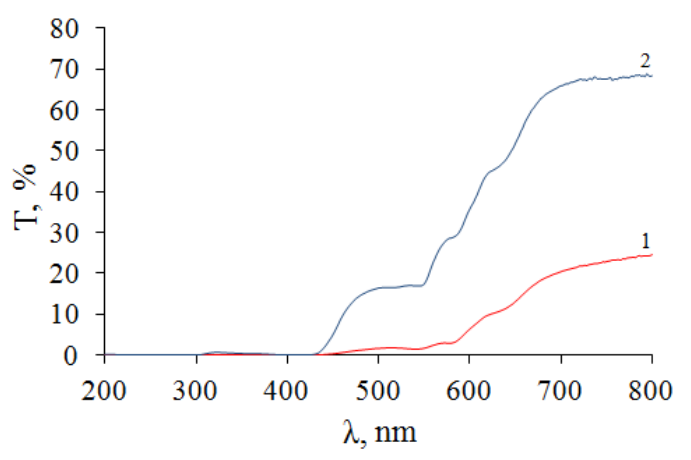

(a)

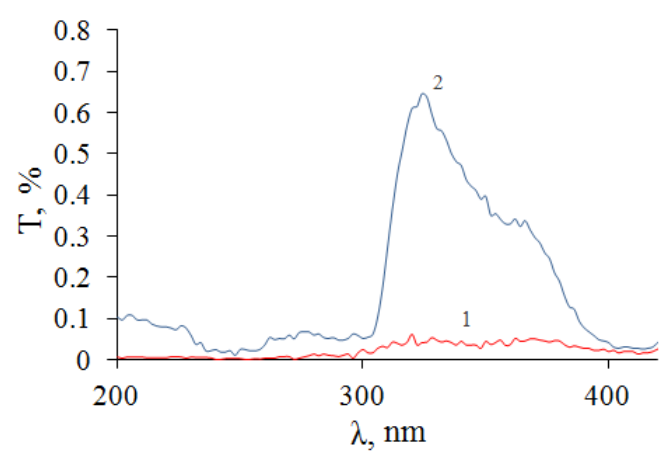

(b)

Fig. 1 TTS before (1) and after (2) diffusion of $87.5 \%$ glycerol solution for $100 \mathrm{~min}$ into a pig gingival sample, in the wavelength range: $200-800 \mathrm{~nm}$ (a); 200-400 nm (b).
In the regions $415-420 \mathrm{~nm}$ and 540-580 nm, dips observed on the TTS correspond to the absorption bands of oxyhemoglobin $(415,542$ and $576 \mathrm{~nm})$. The absorption of water in the measured range of $200-800 \mathrm{~nm}$ is negligible [17]. Glycerol is a highly effective agent for OC of biological tissues. Glycerol is a strong hygroscopic agent that first draws water out of a gingival specimen through osmosis. Then glycerol penetrates into the gingival sample, binds interstitial and intercellular water and increases the refractive index of interstitial space and cytoplasm, providing refractive index matching of scatterers (collagen and elastin fibers) and surrounding media [4]. In Fig. 2, photos of a pig gingival tissue sample before (Fig. 2a) and after (Fig. 2b) immersion in $87.5 \%$ glycerol solution and the diagram for OC efficiency at different wavelengths (Fig. 2c) calculated from experimental data using formula (1) are shown.

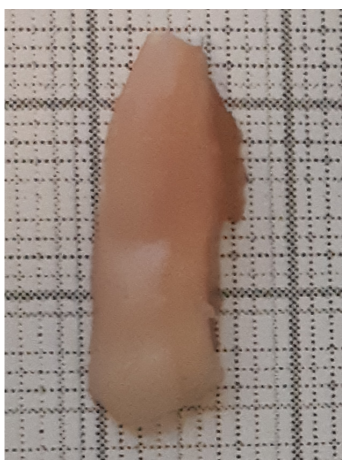

(a)

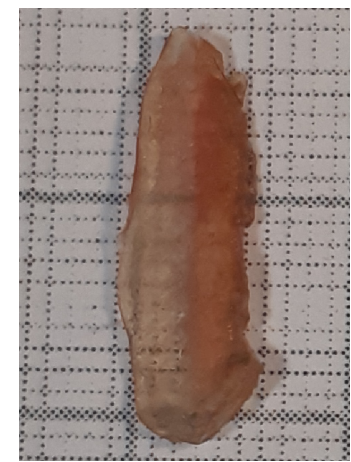

(b)

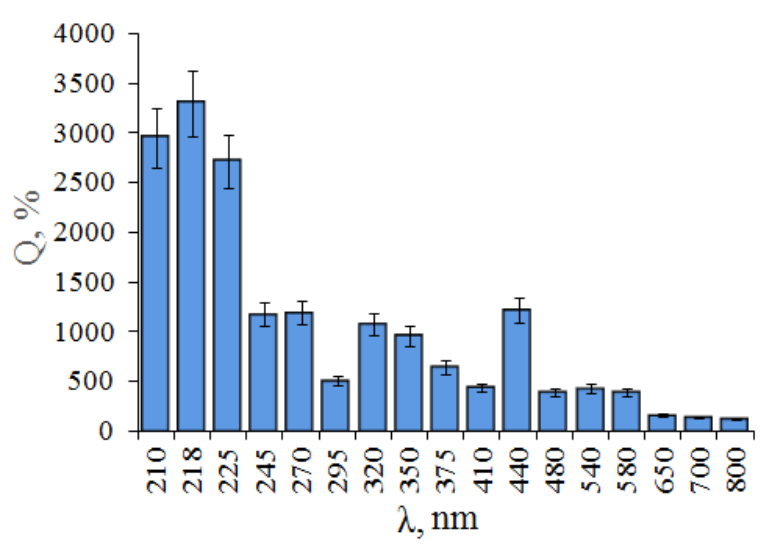

(c)

Fig. 2 Photos of a porcine alveolar gingival sample before (sample size $20.0 \times 6.0 \mathrm{~mm}^{2}$ ) (a) and after (sample size $19.6 \times 5.7 \mathrm{~mm}^{2}$ ) (b) immersion in $87.5 \%$ glycerol solution for $100 \mathrm{~min}$; OC efficiency averaged for gingival samples after $100 \mathrm{~min}$ immersion in $87.5 \%$ glycerol solution calculated from experimental data using Eq. (1).

It follows that the greatest efficiency is achieved at a wavelength of $210-230 \mathrm{~nm}$ for $100 \mathrm{~min}$ of glycerol action and amounts to $3500 \%$. Thus, the efficiency of OC is higher, the lower the initial intensity before OC. On average, the efficiency changes in accordance with the 
behavior of the transmission spectrum (Fig. 2c). In the visible and NIR, within the so-called 'transparent window' [1], the $\mathrm{Q}$ is significantly lower, around 100-200\%; however, due to the absence of strong absorption bands of endogenous chromophores in this spectral region, the absolute transmission is quite large and reaches $60 \%$.

The applied OC treatments with highly concentrated glycerol have induced two tissue windows in the UV range in colorectal tissues - from 200 to $260 \mathrm{~nm}$ and from 260 to $418 \mathrm{~nm}$, it was used to identify pathological tissue [18].

Three dynamic windows were identified in the gingiva mucosa (from 200 to $250 \mathrm{~nm}$, from 250 to 300 $\mathrm{nm}$ and from 300 to $400 \mathrm{~nm}$ ), it we used to increase the effectiveness of the treatment of chronic recurrent aphthous stomatitis (CRAS) in children.

\subsection{Clinical study}

The development of phototherapy may be of particular importance in case of such a common dental disease as CRAS, characterized by a relapsing course with inflammatory and destructive lesions of the oral mucosa. CRAS is a fairly common disease in dental practice. It is characterized by a long course, a tendency to relapse and resistance to various types of therapy [19]. Despite the fact that the nature of the occurrence of this dental disease is still not entirely clear, relapses of CRAS are often associated with some provoking factors, including an infectious nature. There is a neurogenic, immune, infectious-allergic theory of the origin of CRAS, the importance of gastrointestinal pathology, endocrine abnormalities, as well as immunological reactivity and resistance of the body [20].

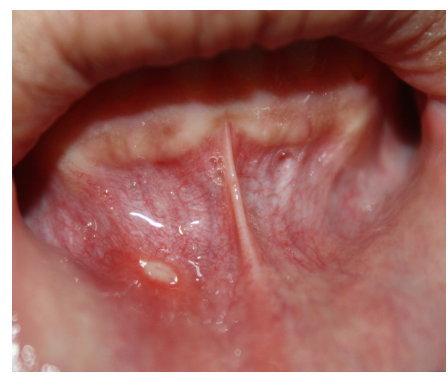

a

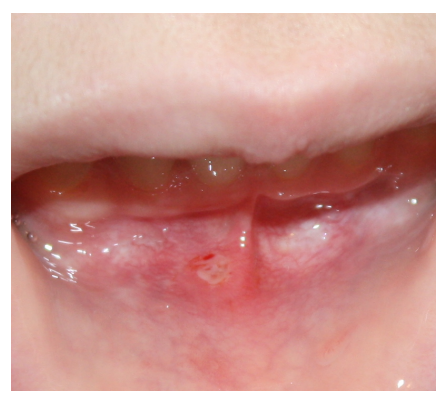

d

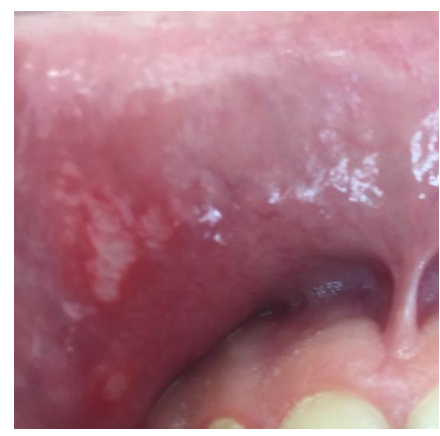

g

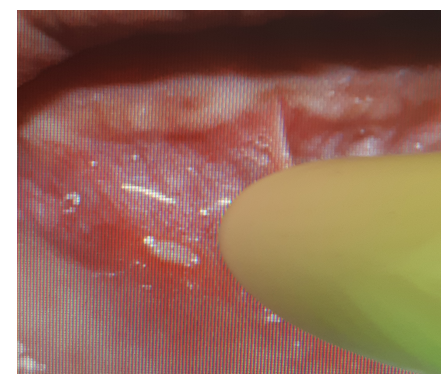

b

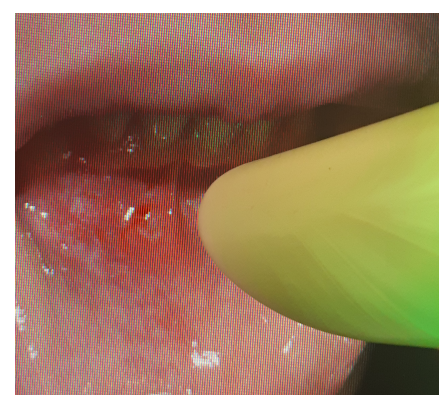

e

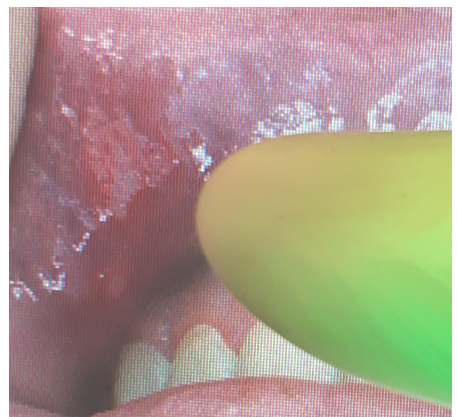

$\mathrm{h}$

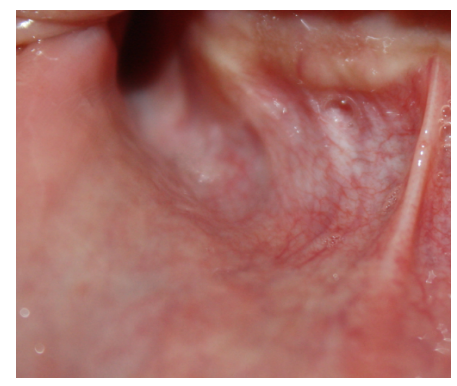

$\mathrm{c}$

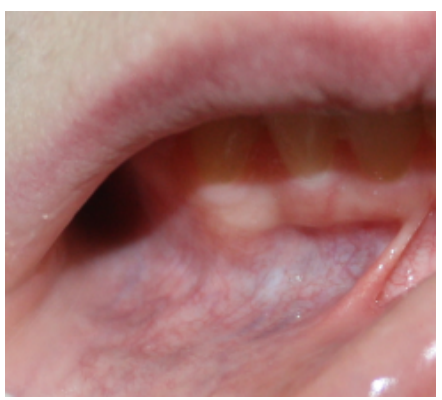

f

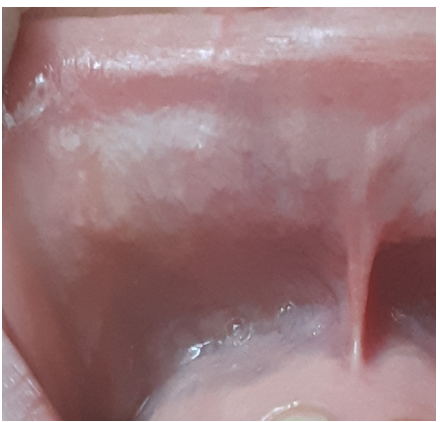

i

Fig. 3 Three examples of stomatitis phototherapy (photo real size): before treatment (a), (d), and (g); OC of the oral mucosa with $87.5 \%$ glycerol solution and subsequent irradiation with UV (b), (e), and (h); and final treatment results (c), (f), and (i). 
In the initial state, the examined patients showed various subjective and objective clinical manifestations of CRAS. The predominant complaint was pain in the area of the affected areas of the mucous membrane, which was aggravated by eating, talking and reducing the quality of life. Quite often, patients were disturbed by a burning sensation in the mouth $(84.5 \%)$, increased salivation $(76.3 \%)$, which forced patients to disrupt their diet, which caused an exacerbation of concomitant chronic diseases.

After applying the full course of phototherapy with the use of the OC with glycerol, relief of the main clinical manifestations of inflammation: complete absence of burning and increased salivation, was clearly observed in $93.6 \%$ of patients. In the comparison and control groups, similar dynamics was observed in $81.1 \%$ and $64.5 \%$, respectively. Dental examination objectively confirmed the complete regression of clinical symptoms in the vast majority of patients in the main group, which is significantly more significant than in the comparison group and, especially, in the control group. Improvement of the subjective state of the observed patients is also associated with the relief of the main signs of local inflammation of the mucous membrane, objectively detected during a dental examination and assessment of the peripheral blood picture.

A pronounced therapeutic efficiency of the developed method $(93.7 \%)$ was revealed in comparison with only UV irradiation $(81.2 \%)$, as well as with standard medicinal dental treatment $(65.9 \%)$.

\section{Conclusions}

At immersion of mucous membrane of the gingiva in glycerol solution, it was found that the highest efficiency of optical clearing occurs in the UV region with the formation of three transparency optical windows: I - from 200 to $250 \mathrm{~nm}$, II - from 250 to $300 \mathrm{~nm}$ and III - from 300 to $400 \mathrm{~nm}$. It is known that UV radiation has great biological activity, antibacterial and immunestimulating effects. For the first time, we clinically applied optical clearing with $87.5 \%$ glycerol solution to increase the penetration depth of UV radiation to treat more efficiently chronic recurrent aphthous stomatitis (CRAS) in children. The conducted observations have shown the effectiveness of this method, its simplicity, and availability, which allow us to recommend it for a widespread use in dentistry in the complex treatment of CRAS and for the prevention of CRAS in patients at risk.

\section{Disclosures}

All authors declare that there is no conflict of interests in this paper.

\section{Acknowledgements}

The authors are grateful to engineer Galina N. Naumova for the help in conducting measurements.

V. V. Tuchin acknowledges the financial support from grant of the Government of the Russian Federation for state support of scientific research conducted under supervision of leading scientists in Russian institutions of higher education, scientific foundations and state research centers of the Russian Federation (registration number 2020-220-08-2389).

\section{References}

1. V. V. Tuchin, Tissue Optics: Light Scattering Methods and Instruments for Medical Diagnostics, SPIE Press, Bellingham (2015).

2. C. Sicora, A. Szilárd, L. Sass, E. Turcsányi, Z. Máté, and I. Vass, "UV-B and UV-A Radiation Effects on Photosynthesis at the Molecular Level," Chapter in: Environmental UV Radiation: Impact on Ecosystems and Human Health and Predictive Models. Nato Science Series: IV: Earth and Environmental Sciences, F. Ghetti, G. Checcucci, J. F. Bornman (eds), Springer, Dordrecht, 121-135 (2006).

3. L. Shi, R. R. Alfano, Deep Imaging in Tissue and Biomedical Materials: Using Linear and Nonlinear Optical Methods, CRC Press, Boca Raton (2017).

4. L. Oliveira, V. V. Tuchin, The Optical Clearing Method: A New Tool for Clinical Practice and Biomedical Engineering, Basel, Springer Nature Switzerland AG (2019).

5. B. Baumann, "Polarization sensitive optical coherence tomography: a review of technology and applications," Applied Sciences 7(5), 474 (2017).

6. Z. Hamdoon, W. Jerjes, G. McKenzie, A. Jay, and C. Hopper, "Optical coherence tomography in the assessment of oral squamous cell carcinoma resection margins," Photodiagnosis and Photodynamic Therapy 13, 211-217 (2016).

7. N. M. Le, Sh. Song, H. Zhou, J. Xu, Y. Li, C.-E. Sung, A. Sadr, K.-H. Chung, H. M. Subhash, L. Kilpatrick, and R. $\mathrm{K}$. Wang, "A noninvasive imaging and measurement using optical coherence tomography angiography for the assessment of gingiva: An in vivo study," Journal of Biophotonics 11(12), e201800242 (2018).

8. M.-T. Tsai, Y. Chen, Ch.-Y. Lee, B.-H. Huang, N. H. Trung, Y.-J. Lee, and Y.-Li Wang, "Noninvasive structural and microvascular anatomy of oral mucosae using handheld optical coherence tomography," Biomedical Optics Express 8(11), 5001-5012 (2017). 
9. M. M. Perez, R. Ghinea, L. J. Herrera, F. Carrillo, A. M. Ionescu, and R. D. Paravina, "Color difference thresholds for computer-simulated human gingival," Journal of Esthetic and Restorative Dentistry 30(2), E24-30 (2018).

10. I. Sailer, "Threshold values for the perception of color changes in human teeth," The International Journal of Periodontics \& Restorative Dentistry 36(6), 777-783 (2016).

11. N. D. Sarmast, N. Angelov, R. Ghinea, J. Powers, and R. Paravina, "Color compatibility of gingival shade guides and gingiva-colored dental materials with healthy human gingival," The International Journal of Periodontics \& Restorative Dentistry 38(3), 397-403 (2018).

12. D. K. Ho, R. Ghinea, L. J. Herrera, N. Angelov, and R. D. Paravina, "Color range and color distribution of healthy human gingiva: a prospective clinical study," Scientific Reports 5(1), 18498 (2015).

13. V. V. Tuchin, Optical Clearing of Tissues and Blood, SPIE Press, Bellingham, USA (2005).

14. H. Kang, C. L. Darling, and D. Fried, "Use of an optical clearing agent to enhance the visibility of subsurface structures and lesions from tooth occlusal surfaces," Journal of Biomedical Optics 21(8), 081206 (2016).

15. J. B. Schutt, J. F. Arens, C. M. Shai, and E. Stromberg, "Highly Reflecting Stable White Paint for the Detection of Ultraviolet and Visible Radiations," Applied Optics 13(10), 2218-2221 (1974).

16. Refractive Index Database (accessed 14 December 2020).

17. A. Vogel, V. Venugopalan, "Mechanisms of pulsed laser ablation of biological tissues," Chemical Reviews 103(2), 577-644 (2003).

18. I. Carneiro, S. Carvalho, R. Henrique, L. Oliveira, and V. Tuchin, "Moving tissue spectral window to the deepultraviolet via optical clearing," Journal of Biophotonics 12(12), e201900181 (2019).

19. N. Altenburg, N. El-Haj, C. Micheli, M. Puttkammer, M. Abdel-Naser, and C. C. Zouboulis, "The Treatment of Chronic Recurrent Oral Aphthous Ulcers," Deutsches Aerzteblatt Online 111(40), 665-673 (2014).

20. B. I. Tarakji, G. Gazal, S. Ali Al-Maweri, S. N. Azzeghaiby, and N. Alaizari "Guideline for the Diagnosis and Treatment of Recurrent Aphthous Stomatitis for Dental Practitioners," Journal of International Oral Health 7(5), 7480 (2015). 